\title{
Polar stratospheric cloud observations by MIPAS on ENVISAT: detection method, validation and analysis of the northern hemisphere winter $2002 / 2003$
}

\author{
R. Spang ${ }^{1}$, J. J. Remedios ${ }^{2}$, L. J. Kramer ${ }^{2}$, L. R. Poole ${ }^{3}$, M. D. Fromm ${ }^{4}$, M. Müller ${ }^{5}$, G. Baumgarten ${ }^{6}$, and \\ P. Konopka ${ }^{1}$ \\ ${ }^{1}$ ICG I, Research Centre Jülich, Germany \\ ${ }^{2}$ EOS/SRC, Department of Physics and Astronomy, University of Leicester, UK \\ ${ }^{3}$ Atmospheric Science Competency, NASA Langley Research Center, Hampton, Virginia, USA \\ ${ }^{4}$ Computational Physics, Incorporated, Springfield, Virginia, USA \\ ${ }^{5}$ Alfred Wegener Institute, Potsdam, Germany \\ ${ }^{6}$ Leibniz-Institute of Atmospheric Physics, Kühlungsborn, Germany
}

Received: 26 July 2004 - Published in Atmos. Chem. Phys. Discuss.: 6 October 2004

Revised: 18 January 2005 - Accepted: 18 February 2005 - Published: 2 March 2005

\begin{abstract}
The Michelson Interferometer for Passive Atmospheric Sounding (MIPAS) on ENVISAT has made extensive measurements of polar stratospheric clouds (PSCs) in the northern hemisphere winter 2002/2003. A PSC detection method based on a ratio of radiances (the cloud index) has been implemented for MIPAS and is validated in this study with respect to ground-based lidar and space borne occultation measurements. A very good correspondence in PSC sighting and cloud altitude between MIPAS detections and those of other instruments is found for cloud index values of less than four. Comparisons with data from the Stratospheric Aerosol and Gas Experiment (SAGE) III are used to further show that the sensitivity of the MIPAS detection method for this threshold value of cloud index is approximately equivalent to an extinction limit of $10^{-3} \mathrm{~km}^{-1}$ at $1022 \mathrm{~nm}$, a wavelength used by solar occultation experiments. The MIPAS cloud index data are subsequently used to examine, for the first time with any technique, the evolution of PSCs throughout the Arctic polar vortex up to a latitude close to $90^{\circ}$ north on a near-daily basis. We find that the winter of 2002/2003 is characterised by three phases of very different PSC activity. First, an unusual, extremely cold phase in the first three weeks of December resulted in high PSC occurrence rates. This was followed by a second phase of only moderate PSC activity from 5-13 January, separated from the first phase by a minor warming event. Finally there was a third phase from February to the end of March where only sporadic and mostly weak PSC events took place. The compo-
\end{abstract}

Correspondence to: R. Spang

(r.spang@fz-juelich.de) sition of PSCs during the winter period has also been examined, exploiting in particular an infra-red spectral signature which is probably characteristic of NAT. The MIPAS observations show the presence of these particles on a number of occasions in December but very rarely in January. The PSC type differentiation from MIPAS indicates that future comparisons of PSC observations with microphysical and denitrification models might be revealing about aspects of solid particle existence and location.

\section{Introduction}

Despite progress in the observation, modelling and understanding of polar stratospheric clouds (PSCs) in recent years, there are still important questions which remain to be resolved, particularly with respect to the microphysical formation of solid nitric-acid-containing particles such as nitric acid trihydrate (NAT). Recently, the presence of NAT in the stratosphere has been definitively confirmed (Voigt et al., 2002) and the range of possible NAT particle sizes extended to large radii (typically $5-10 \mu \mathrm{m}$ ) as inferred by Fahey et al. (2001). However, the nucleation mechanism for NAT particles remains unknown, although a large number of possible mechanisms have been suggested (Peter, 1997; Tabazadeh et al., 2001). This is important since solid particles, particularly NAT particles, are believed to contribute significantly to denitrification in a number of Arctic winters (e.g. Fahey et al., 2001; Carslaw et al., 2002; Mann et al., 2002) and subsequent ozone loss. It is clear that in order to distinguish NAT formation processes and to validate

(C) 2005 Author(s). This work is licensed under a Creative Commons License. 
microphysical and denitrification models, it would be highly beneficial to improve continuous monitoring of PSC cloud coverage and, where possible PSC composition, on a vortexwide basis. Spaceborne sensors can provide an essential view of PSC evolution in both polar vortices and, in conjunction with satellite measurements of nitric acid concentrations, deliver a better understanding and quantification of denitrification.

Since the end of the 1970s, observations of PSCs by spaceborne occultation and emission instruments have been published frequently (e.g. McCormick et al., 1982; Taylor et al., 1994; Massie et al., 1998; Spang et al., 2001). The most recent instruments provide significantly enhanced capabilities for the detection of PSCs including one or more of the following features: broadband and high spectrally resolved measurements of PSCs, dedicated latitude coverage or narrow fields-of-view (FOV). In this paper, we present new results from the Michelson Interferometer for Passive Atmospheric Sounding (MIPAS) from the Arctic winter 2002/2003. The MIPAS instrument provides infra-red (IR) spectra of PSCs at high spectral resolution with unprecedented latitude coverage of up to nearly $90^{\circ}$ south and north. Locations of PSCs in the Antarctic and Arctic polar vortices can be tracked daily and indications of PSC type obtained from the data. The analyses conducted in support of the major Vintersol/SOLVE II campaign, which took place during winter 2002/2003 in the Arctic, are described in this paper.

The paper is structured as follows. Sections 2 and 3 give an overview of the instrument and the cloud detection and type differentiation method, followed by a detailed validation against PSC observations from ground-based lidar and spaceborne solar occultation instruments in Sect. 4. Section 5 describes the temporal and geographical distribution of the PSC occurrence with respect to the evolution of the polar vortex.

\section{The MIPAS instrument}

On 1 March 2002, the Michelson Interferometer for Passive Atmospheric Sounding (MIPAS) was launched on the ENVISAT platform. It is a Fourier transform spectrometer providing limb sounding spectra of atmospheric infrared emission between $685 \mathrm{~cm}^{-1}(14.60 \mu \mathrm{m})$ and $2410 \mathrm{~cm}^{-1}$ $(4.15 \mu \mathrm{m})$ at a spectral unapodised resolution of $0.025 \mathrm{~cm}^{-1}$ (Fischer and Oelhaf, 1996). The high spectral resolution enables for a better separation to be made between the line emission of the atmospheric trace gases and continuum emission from PSCs and tropospheric clouds compared to the moderate resolution IR instruments such as the CRyogenic Infrared Spectrometers and Telescopes for the Atmosphere (CRISTA) instrument (Riese et al., 1997). With MIPAS, vertical profiles are scanned from $6 \mathrm{~km}$ to $68 \mathrm{~km}$ with a vertical spacing of $3 \mathrm{~km}$ in the lower stratosphere, commensurate with the field-of-view of the instrument and hence its nominal vertical resolution.

The PSC information is extracted from the MIPAS level $1 \mathrm{~b}$ product, which consists of calibrated IR radiance spectra. The calibration is based on views of a warm blackbody $(238 \mathrm{~K})$, cold space ( $235 \mathrm{~km}$ tangent height) and a correction for non-linearity of the detectors. In this study, we utilise near-real-time (NRT) data for MIPAS processed by the ESA NRT operational processor with software versions 4.53 to 4.57 (November 2002-December 2003).

Validation studies indicate that the offset along one orbit is less than $6 \mathrm{nW} / \mathrm{cm}^{-2} \mathrm{sr} \mathrm{cm}^{-1}$ (personal communication: A. Kleinert, IMK, Research Centre Karlsruhe) and tests show that the offset error in the calibrated spectra induced by this variation is less than $2-3 \mathrm{nW} / \mathrm{cm}^{-2} \mathrm{sr} \mathrm{cm}^{-1}$. Not all the desirable MIPAS level $1 \mathrm{~b}$ datasets were available for this analysis, which was performed at the time of the Vintersol campaign. However, the available data are sufficient to demonstrate the key characteristics of the observed PSC distribution during the winter studied and to identify the important spatial variations of the PSCs.

\section{MIPAS cloud information}

\subsection{Cloud detection method}

The occurrence of clouds, such as PSCs, in the field-of-view of a limb-scanning instrument produces a characteristic enhancement in the broadband continuum radiation component of the measured infra-red spectra. This enhancement can be detected with a relatively coarse spectral resolution $\left(2 \mathrm{~cm}^{-1}\right)$ as was shown for the CRISTA instrument (for example, Spang and Remedios, 2003). We therefore follow the experience of the CRISTA cloud analyses (Spang et al., 2002) in the cloud detection for MIPAS. This is accomplished by using the ratio of radiances in the $788-796 \mathrm{~cm}^{-1}$ range, dominated by $\mathrm{CO}_{2}$, and the $832-834 \mathrm{~cm}^{-1}$ range in the atmospheric window regions, dominated by aerosol and clouds emissions (Fig. 1). The latter region contains only weak emissions of ozone and CFC11 in comparison to the background aerosol and the enhanced cloud radiation. Both wavelength regions are in the MIPAS detector band A $\left(685-970 \mathrm{~cm}^{-1}\right)$ and in the following the defined ratio will be referred to as the cloudindex (CI). The method has been successfully applied to the MIPAS measurements (Remedios and Spang, 2002; Spang et al., 2004) and is in the process of implementation and validation in ESA's operational MIPAS retrieval processor; the operational implementation includes cloud index derivations for MIPAS bands A, B and D (Remedios and Spang, 2003) but only CI profiles for band A are used here.

In principle, retrieved extinction profiles would be most useful for the detection of PSCs. This approach works quite well for solar occultation experiments in the visible and nearIR but is much more difficult for IR-emission instruments 


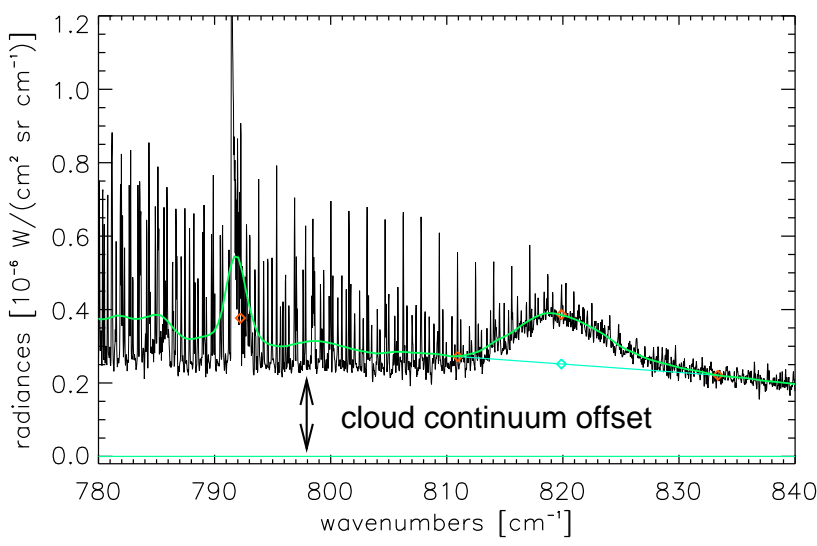

Fig. 1. An apodised MIPAS spectrum (in black) of an optically thin PSC with a characteristic spectral signature around $820 \mathrm{~cm}^{-1}$ measured on 11 December 2002 08:09 UT at 69.9 $\mathrm{N}$ and 32.8 $\mathrm{E}$ in the northern hemisphere polar vortex. A curve showing a spectrum with reduced spectral resolution similar to CRISTA is superimposed in green. The red symbols present the mean radiances used for cloud detection and type differentiation (details see text).

due to problems in representing the mixed continuum and trace gas contributions when scattering is important. Höpfner et al. (2002) have shown that the scattering of tropospheric radiation by PSCs can become significant for particle radii $>1 \mu \mathrm{m}$. Modelling of such clouds in the IR is then not only prohibitively expensive for routine retrievals but also it is often hampered by a lack of knowledge of source terms such as the underlying tropospheric clouds. The radiancebased cloud detection method employed here has the big advantage that no time-consuming retrieval process is necessary. It delivers a qualitative measure of the optical thickness of the cloud and allows a robust detection of PSCs.

Typical CI profiles for MIPAS are shown in Fig. 2 for profiles for which a $\mathrm{CI}>4$ does not indicate PSCs (black profiles) and for profiles with PSCs (red profiles). The radiance ratio decreases rapidly from one altitude step to the next from background values of around 6-10 to 1-4, if a cloud is in the field-of-view. Constant values of CI close to unity indicate optically thick conditions. Sensitivity studies for CI with the CRISTA forward radiance model and the MIPAS reference forward model (RFM) were carried out and reported in Spang et al. (2004) and references therein. The temperature dependence of CI was shown to be especially weak (less than $1 \% / \mathrm{K}$ in the 10 to $30 \mathrm{~km}$ range), so that a single threshold value is sufficient for the PSC study here.

In MIPAS operational processing, a conservative threshold of 1.8 has been implemented, which guarantees the detection of strong cloud events over relatively broad height range of $12-40 \mathrm{~km}$. The clouds identified with this threshold value are those which are most likely to affect the retrieval of trace gases from MIPAS data. For the detection of PSCs we extended the threshold value to 4 but limited the height region

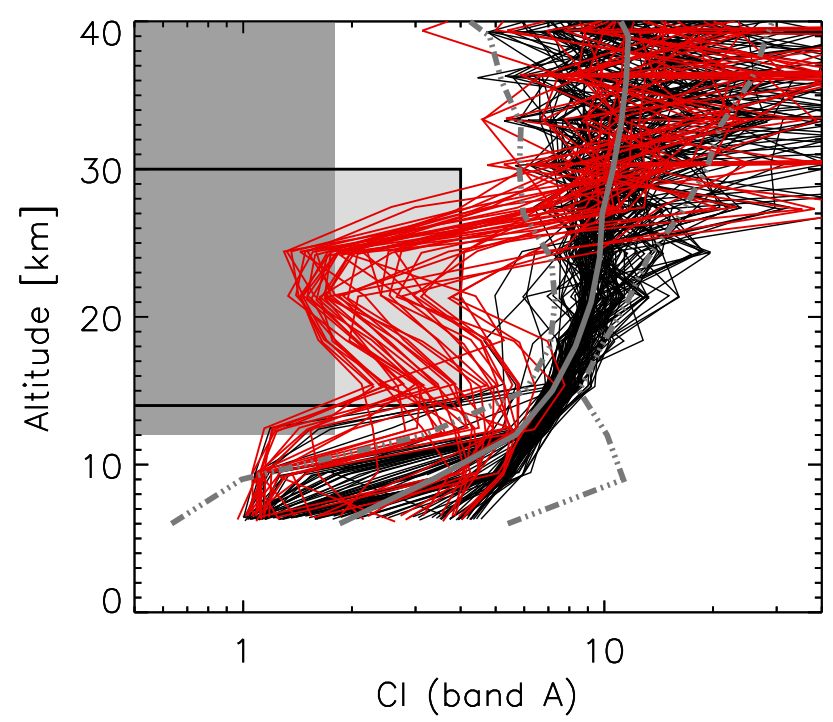

Fig. 2. Cloud index profiles for the MIPAS wavelength band $A$ in the time frame 1 to 11 December in the latitude band $75^{\circ} \mathrm{N}$ to $80^{\circ} \mathrm{N}$ (total 174). Red curves are highlighting profiles which are marked as 'cloudy' by a CI threshold value of $\mathrm{CI}<4$ above $14 \mathrm{~km}$. Shaded box areas indicate (a) the cloudy area in the CI height space for the MIPAS operational processor (dark grey), (b) the threshold values used in the analyses below (grey plus dark grey, framed by black line). Red curves highlight profiles which are marked as 'cloudy' by a CI threshold value of $\mathrm{CI}<4$ above $14 \mathrm{~km}$. Shaded box areas indicate (a) the cloudy area in the CI-height-space for the MIPAS operational processor (dark grey) with $\mathrm{CI}<1.8$ and altitudes between 12 and $40 \mathrm{~km}$, (b) the threshold values used in the analyses below (grey plus dark grey, framed by black line) with $\mathrm{CI}<4$ and altitude range 14 to $30 \mathrm{~km}$. A mean of PSC-free profile between $50^{\circ}-55^{\circ} \mathrm{N}$ of $\log (\mathrm{CI})$ and $\log (\mathrm{CI})+/-2 \times$ standard deviation of $\log (\mathrm{CI})$ (dashdotted) are superimposed in thick grey.

to 14 to $30 \mathrm{~km}$. At this threshold level, optically thinner cloud events are detectable at levels which are still in excess of the noise level; at this threshold CI values at $15 \mathrm{~km}$ should still be treated with caution due to the relatively coarse FOV of MIPAS and potential effects by high tropospheric clouds.

The analysis also allows us to approximate a cloud top height $(\mathrm{CTH})$ by computing the first tangent height where CI falls below the specified threshold. Essentially, this indicates the highest tangent altitude for MIPAS at which a PSC is observed in one vertical scan. Cloud effects at the tangent heights below $\mathrm{CTH}$ are difficult to interpret due to the mixing of emission from clouds at the actual tangent height with emission from the layers of the limb path above. Increasing CI values below the $\mathrm{CTH}$, as commonly observed for almost all PSC profiles in this study, provide indirect and approximate information about the optical thickness and vertical extent of the cloud since they would be consistent with an increasing thickness of PSCs if the cloud field is homogeneous. An optically thick cloud would generate a CI value close to one. Due to the blocking effect of this layer, the 

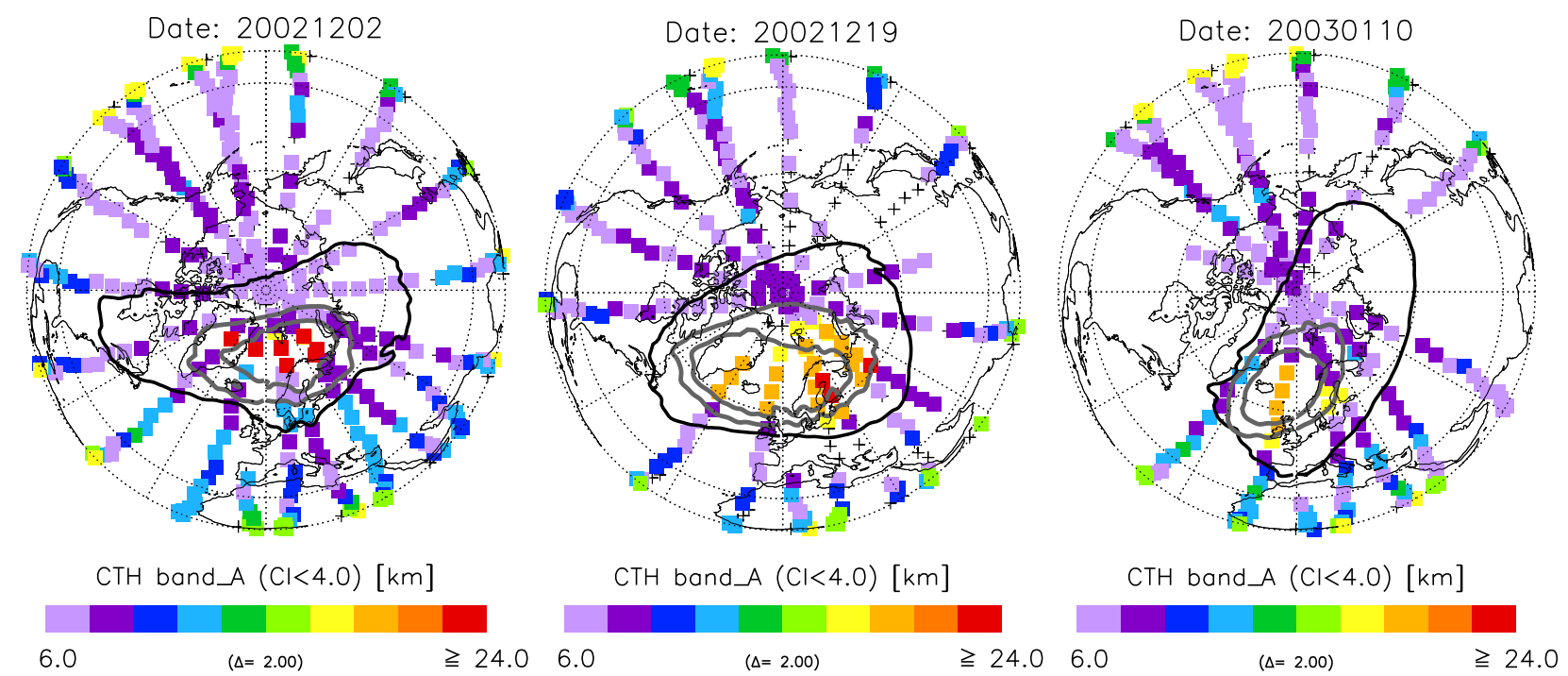

Fig. 3. Northern polar projection of CTH observations $(\mathrm{CI}<4)$ by MIPAS for 2 December, (the first day of distinctive PSC occurrence) 19 December and 10 January. Cloud-free profiles down to the minimum tangent height of around $6 \mathrm{~km}$ are marked by crosses. The black line highlights the vortex boundary (Nash-criterion) and the grey contour pair belongs to $\mathrm{T}_{-} \mathrm{T}_{N A T}=0 \mathrm{~K}$ and $-3 \mathrm{~K}$, respectively, where $\mathrm{T}_{N A T}$ is calculated with constant $10 \mathrm{ppbv} \mathrm{HNO}_{3}$ and 5 ppmv $\mathrm{H}_{2} \mathrm{O}$ ). The contours are for the $575 \mathrm{~K}$ isentrope with the exception of $10 \mathrm{January}$ for the $525 \mathrm{~K}$ isentrope.

CI values would stay constant at all the tangent heights below the first optically thick layer. For an optically thin cloud, CI values will increase for tangent heights below the altitude which contains the largest optical thickness (simply by the limb path). However, generally the CI values do not reach cloud-free values again, because the limb path is still passing through the cloud layers even for the tangent height layers below the cloud bottom. Therefore the vertical extent suggested by the CI profile is generally overestimated and an accurate determination is not achievable by the simple colour ratio method.

Examples of resulting maps of CTHs for 2 and 19 December 2002 and 10 January 2003 are given in Fig. 3 (details in Sect. 5.2). 2 December shows the first day of distinctive PSC occurrence in the MIPAS observations (weak PSC activity is also observed on 1 December). Lower CTHs can be observed later in the season (Fig. 3c). The absolute pointing error of the MIPAS instrument and the subsequent CTH error is estimated to be of the order of $1-1.5 \mathrm{~km}$. Unfortunately the pointing error drifts with time and increases for high northern latitudes. Tangent heights retrieved by the method of von Clarmann et al. (2004) show (+)0.2 km higher altitudes in November 2002 than the so-called MIPAS engineering heights of the ESA NRT product. Until March the difference changes nearly linearly to (-) $1.7 \mathrm{~km}$ lower altitudes (M. Kiefer, Research Centre Karlsruhe, personal communication). For December data in particular, the error should be smaller $(<500 \mathrm{~m})$ than the width of the field-of-view. Finally, the relatively broad FOV of the instrument is an additional error source for CTH determination $( \pm 1.5 \mathrm{~km}$ for optically thick clouds). For thinner clouds, which need some coverage of the FOV to be detected, the error becomes asymmetrical around the tangent height and MIPAS detects systematically lower clouds.

\subsection{PSC type differentiation method}

A large subset of the MIPAS PSC spectra show a characteristic spectral feature at $820 \mathrm{~cm}^{-1}$ (Fig. 1), $+/-10 \mathrm{~cm}^{-1}$ width, which has been analysed by Spang and Remedios (2003) using the CRISTA data. The authors showed that the observed features are most likely related to NAT clouds with particle size distributions of small and moderate mean radii $(<2-3 \mu \mathrm{m})$. Those clouds which do not show the spectral feature and are not optically thick are most likely to be clouds of liquid sulfuric ternary solution droplets (STS, PSC type 1b), mixed clouds dominated by STS, or clouds of larger NAT particles. For a better differentiation between the so-called radiance-enhanced (RE) events at $820 \mathrm{~cm}^{-1}$ and those events which are not radiance-enhanced (NRE), we computed the percentage radiance enhancement of the mean $818.3-821.45 \mathrm{~cm}^{-1}$ emission in relation to an estimated background value at $820 \mathrm{~cm}^{-1}$. The latter term is computed by a simple interpolation between the mean radiances at the neighbouring wavenumber regions 810.25$811.65 \mathrm{~cm}^{-1}$ and $832.3-834.4 \mathrm{~cm}^{-1}$ (see Fig. 1). A visual check of the spectra detected by this method gives us confidence that the method works properly and an enhancement 
of greater than $10 \%$ has been defined for a clear signature in the continuum-like part of the MIPAS spectrum around $820 \mathrm{~cm}^{-1}$. For example, the spectrum in Figure 1 shows an extremely large enhancement of 35\%. Such strong RE events have been observed in many CRISTA PSC spectra in the southern polar vortex (Spang and Remedios, 2003, Fig. 2a) but not so frequently in MIPAS observations of the northern hemisphere.

\section{Validation results}

For winter 2002/2003, a number of observations of PSCs are available for comparison with the detections of PSCs from MIPAS using our analysis method. We compare here two ground-based lidar instruments and satellite occultation instruments which provide reasonable statistics for coincidences with the MIPAS data. Two factors are important. For the validation tests performed here, the CI threshold was set to 4 , consistent with the possibilities discussed in Sect. 3.1. In addition, a visual check for each coincidence was performed to exclude potential misinterpretations of the CI profiles. Secondly, each coincidence between a measurement by MIPAS and a measurement by another instrument is characterised by a miss-distance and a miss-time which are minimised whilst retaining reasonable coincidence statistics.

\subsection{Lidar comparisons}

During winter 2002/2003 several lidar-stations at high northern latitudes observed PSC events quite frequently. We have concentrated the comparisons in this study on the AWI lidar at Ny-Alesund, Spitsbergen $\left(79^{\circ} \mathrm{N}, 12^{\circ} \mathrm{E}\right)$ and the ALOMAR RMR lidar in Northern Norway $\left(69^{\circ} \mathrm{N}, 16^{\circ} \mathrm{E}\right.$ ) (von Zahn et al., 2000). Each lidar operated at three wavelengths $(353 \mathrm{~nm} / 355 \mathrm{~nm}, 532 \mathrm{~nm}, 1064 \mathrm{~nm})$ and measured the depolarisation at $532 \mathrm{~nm}$. As well as PSC detection, these measurements allow the classification of the observations into different PSC types in the traditional manner for lidar (Browell et al., 1990). The classification criteria applied utilise the lidar backscatter ratio at $532 \mathrm{~nm}\left(\mathrm{R}_{532}\right)$ and volume depolarisation. Here liquid STS particles show no depolarisation whereas both ice and NAT particles show depolarisation but are separated by the strong $\mathrm{R}_{532}>10$ for ice particles. Coincidences were calculated and analysed on a statistical basis for the December/January period for both lidar stations. For all measurement periods of the lidar stations, coincidences with MIPAS measurements were computed. The number of coincident MIPAS and lidar measurements is limited (1) by the number of lidar measurements, which are occasionally hampered by bad tropospheric weather conditions and (2) by the reduced temporal and geographical coverage of MIPAS data currently available for winter 2002/2003. Nevertheless, for cloudy and non-cloudy lidar conditions for both stations 55
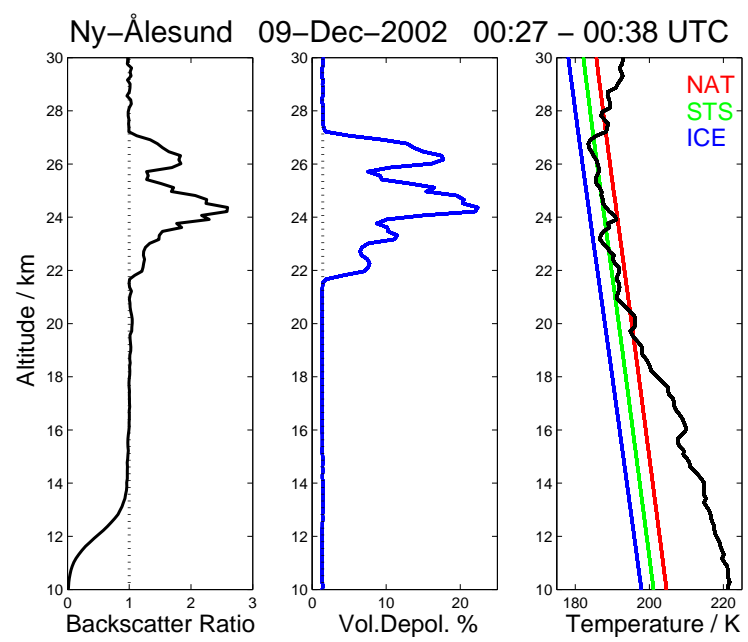

(a)
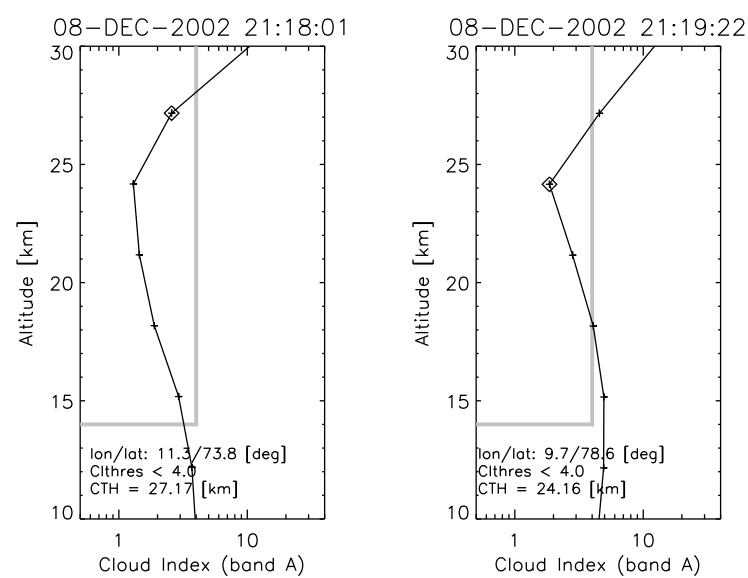

(b)

Fig. 4. (a) AWI lidar single profiles of backscatter ratio and volume depolarisation as well as corresponding radiosonde measurements at the station. (b) MIPAS coincidences with a miss-time of $\sim 3 \mathrm{~h}$ and miss-distance of $383 \mathrm{~km}$ (left) and $167 \mathrm{~km}$ (right).

coincidences with MIPAS were found with miss-time $<4 \mathrm{~h}$ and miss-distances $<400 \mathrm{~km}$ (see the summarising Table 1).

Figure 4 shows a single profile comparison between the AWI lidar and two MIPAS profiles around midnight on 8 December 2002. The backscatter ratio indicates a PSC layer between 22 and $27 \mathrm{~km}$. High depolarisation gives clear indications of solid type clouds and the event was classified as a PSC type Ia. The collocated MIPAS profiles show a very good correspondence in the cloud top height. The closer profile (right side of the figure) with $167 \mathrm{~km}$ miss-distance shows only weak indications of PSC particles at around 27 $\mathrm{km}$. However, these are more strongly developed in the $380 \mathrm{~km}$ miss-distance profile on the left side of the figure $(\mathrm{CI}<3$ at $\sim 27 \mathrm{~km})$. The increasing CIs with decreasing altitude for the closer MIPAS profile give the impression of a vertically limited extent of the cloud of around three tangent height layers $(6-9 \mathrm{~km})$, which is only a rough estimate with large uncertainties (see Sect. 3.1). However, for the presented 
Table 1. Summary of validation comparison for miss-time $<4 \mathrm{~h}$, miss-distance $<400 \mathrm{~km}$ and $\mathrm{CI}<4$ for coincidences in December to January 2002/2003.

\begin{tabular}{lcccc}
\hline Station/Satellite & $\begin{array}{c}\text { Both instruments } \\
\text { cloudy }\end{array}$ & $\begin{array}{c}\text { Only Lidar/SAGE/ } \\
\text { POAM cloudy }\end{array}$ & $\begin{array}{c}\text { Only MIPAS } \\
\text { cloudy }\end{array}$ & $\begin{array}{c}\text { Both } \\
\text { non-cloudy }\end{array}$ \\
\hline Ny-Ålesund & 17 & 2 & 0 & 21 \\
ALOMAR & 10 & 1 & 0 & 4 \\
POAM III & 16 & 21 & 3 & 52 \\
SAGE III & 38 & 34 & 1 & 206 \\
\hline
\end{tabular}

coincidence the limited extent of the cloud is confirmed by the vertically resolved lidar PSC measurements.

In general, the MIPAS-lidar comparison gives a nearly perfect match in the detection of cloudy and non-cloudy events (Table 1). Only 3 of 55 coincidences show dissimilar results, hence MIPAS shows sensitivity comparable to that of the lidars for the detection of these PSC events. The good correspondence is probably positively influenced by the relatively large vertical and horizontal extent of the lidar PSC-events and there is evidence that at these lidar locations the whole winter was dominated by synoptic-scale PSC events. Backscatter ratios $>1.1$ were used to derive lidar CTHs and to compare the results with MIPAS. The MIPAS detection method shows a generally good agreement with the Ny Alsesund lidar (mean CTH difference MIPAS - lidar of $-1.3 \mathrm{~km}$, with a standard deviation $\sigma=1.6 \mathrm{~km}$ ), but also indications of a systematic negative bias to the ALOMAR lidar (mean difference $-2.5 \mathrm{~km}$ and $\sigma=0.9 \mathrm{~km}$ ).

\subsection{Satellite-satellite intercomparisons}

Only solar occultation satellite measurements are available for comparison with the MIPAS data during Arctic winter 2002/2003. The comparisons which are performed here with the Polar Ozone and Aerosol Measurement (POAM) III (Bevilacqua et al., 2002) and the Stratospheric Aerosol and Gas Experiment (SAGE) III (Poole et al., 2003) experiments are therefore restricted in latitude and temporal coverage within the polar vortex by the requirements for sunrise/sunset geometry of these instruments.

\subsubsection{MIPAS-POAM III coincidences}

POAM III is a solar occultation experiment that makes measurements mainly at high northern and southern latitudes (Bevilacqua et al., 2002). POAM makes 14-15 profile measurements per day in each hemisphere. The northern hemisphere measurement latitudes varied from about $64^{\circ}$ in early December 2003 and increased monotonically to a maximum of about $68^{\circ}$ in early March 2004. The vertical resolution of POAM III $(1.5-1.9 \mathrm{~km})$ is better than MIPAS, and is improved by a slightly over-sampled vertical spacing of $1 \mathrm{~km}$ between two tangent heights, without any altitude bias for the tangent heights themselves. Due to the orbit geometries of the two satellites, only miss-times of close to 4 and close to $8 \mathrm{~h}$ are possible. For better count statistics and in agreement with the relatively large measurement volume for each instrument (MIPAS: $3 \times 30 \times 400 \mathrm{~km}^{3}$, POAM III: $\sim 1.5 \times 25 \times 250 \mathrm{~km}^{3}$ ) a maximum miss-time of $4.5 \mathrm{~h}$ and a miss-distance of $400 \mathrm{~km}$ were chosen. Figure 5 shows a single profile comparison for a small miss-distance of $123 \mathrm{~km}$ and minimal possible miss-time of $3 \frac{1}{2} \mathrm{~h}$. PSC detection heights in the POAM profile (left side of figure) are highlighted by triangles (POAM Version 3 data); the PSC detection method for POAM is described and validated in Fromm et al. (2003). Briefly, any retrieved aerosol extinction value which is greater than 2.7 standard deviations, higher than a appropriate clear air average, and which occurs at temperatures lower than a threshold value is assumed to be a PSC.

The broad vertical structure of enhanced extinction of 6$7 \mathrm{~km}$ in the POAM III cloud event in Fig. 5 (left) is very well confirmed in the MIPAS CI profile (right). The simple detection method of MIPAS does not allow an easy determination of the vertical extent of the cloud. However, the recovery in $\mathrm{CI}$ to higher values below $21 \mathrm{~km}$ is a clear indication of optically thinner conditions at $18 \mathrm{~km}$ and levels below. The cloud top heights for both profiles are in very good agreement. This is also the case for most of the 16 "good" matches in Table 1. The mean MIPAS CTH is $0.5 \mathrm{~km}(\sigma=1.5 \mathrm{~km})$ higher than for POAM, which compares very well given the wider FOV of MIPAS and its current pointing accuracy.

Although the single profile above shows a very good correspondence between MIPAS and POAM III, the overall statistic for all potential coincidences in Table 1 results in a significantly larger number of PSC profiles detected by POAM. Most of these 21 profiles are characterised by thin layers in the POAM data $(<3-4$ tangent heights layers within 3-4 km vertical extent) of enhanced extinction and/or relatively low extinction enhancement (optically thin clouds), which could be hard to detect by MIPAS. Only five of the 21 coincidences show strong extinction enhancement for POAM and no cloud indications in the MIPAS spectra, which might be due to viewing geometries or spatial inhomogeneities in the PSC locations. 

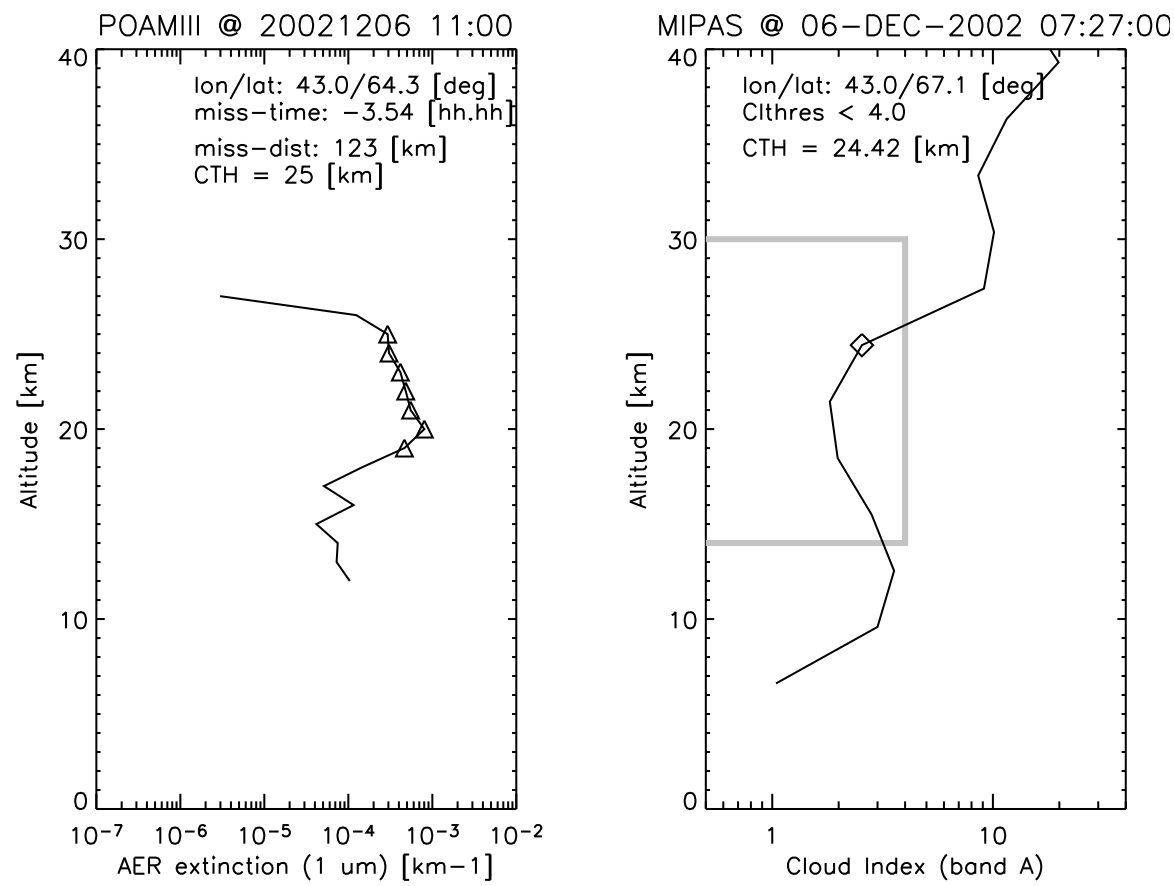

Fig. 5. POAM aerosol extinction profile with PSC altitudes highlighted by triangles (left) versus CI profile of a MIPAS coincidence (right) with miss-time of $3.5 \mathrm{~h}$ and miss-distance of $123 \mathrm{~km}$. MIPAS CTH marked by a diamond.

\subsubsection{MIPAS-SAGE III coincidences}

The SAGE) III instrument has been operating in a sunsynchronous polar orbit onboard the Russian Meteor 3M satellite since late February 2002. SAGE III uses the solar occultation technique to measure $0.5-\mathrm{km}$ vertical resolution profiles of aerosol extinction (with RMS uncertainties) in nine spectral channels ranging in centre wavelength from 384 to $1545 \mathrm{~nm}$. Poole et al. (2003) showed that a two-channel $(449 \mathrm{~nm}$ and $1022 \mathrm{~nm})$ analysis approach enables discrimination of PSC Types 1a and 1b (a method first applied by Strawa et al., 2003 to POAM data) and illustrated that SAGE III is sensitive to mixtures of background aerosol with very few large NAT particles (i.e. NAT rocks).

The approach used to determine SAGE III PSC top altitudes for this paper is similar to Poole et al. (2003), except that data from a third aerosol channel $(1545 \mathrm{~nm})$ were also included to enhance the sensitivity to large particles. SAGE III version 2 data were used for all analyses. Specifically, the analysis used the following SAGE III variables: aerosol extinction at $1022 \mathrm{~nm}\left(\mathrm{e}_{1022}\right)$, the colour ratio of extinctions at $1022 \mathrm{~nm}$ and $449 \mathrm{~nm}\left(\mathrm{CR}_{1}\right)$, and the ratio of extinctions at $1545 \mathrm{~nm}$ and $1022 \mathrm{~nm}\left(\mathrm{CR}_{2}\right)$; data points with $>100 \%$ uncertainty in any of the three variables were excluded. Arctic data for the period November 2002-March 2003 were divided into monthly ensembles, which were partitioned into $50 \mathrm{~K}$ potential temperature bins over the $400-700 \mathrm{~K}$ range. Background aerosol (non-PSC) data points were identified as those with United Kingdom Meteorological Office temperatures of more than $5 \mathrm{~K}$ above the NAT equilibrium temperature (see Poole et al., 2003 for details). For these background aerosol subsets, median values $\left(\operatorname{med}_{i}\right)$ of $\mathrm{e}_{1022}, \mathrm{CR}_{1}$, and $\mathrm{CR}_{2}$ were calculated, as well as mean absolute deviations $\left(\delta_{i}\right)$ about the medians. The PSCs were then identified as those outlying points for which any of the three variables minus its RMS uncertainty exceeded med $_{i}+3 \delta_{i}$ for the particular background aerosol subset. The PSC top altitudes are identified as the maximum altitude at which a data point was identified as a PSC in any SAGE III vertical profile, as with the MIPAS detection method. In addition, PSC top altitude points were classified as Type $1 \mathrm{~b}$ if the value of $\mathrm{e}_{1022}$ was greater than the med $\left(\mathrm{e}_{1022}\right)+3 \delta\left(\mathrm{e}_{1022}\right)$ for the background aerosol ("outlier"), accompanied by only a modest increase in the values of CR1 and CR2 relative to the background aerosol, as would be expected from an STS cloud; as Type 1a if the point was an outlier primarily in either $\mathrm{CR}_{1}$ or $\mathrm{CR}_{2}$; and as type "mixed" if neither Type $1 \mathrm{a}$ or $1 \mathrm{~b}$ could be determined with confidence. It should be mentioned that the differentiation methods described here and by Strawa et al. (2003) are based on the different size distributions of STS and NAT, while that of MIPAS is a real spectroscopic method.

The comparison between MIPAS and SAGE III benefits from a better correspondence in the orbit and observation geometry of both instruments, which results in generally much smaller miss-times ( $<60 \mathrm{~min}$ ) and a significantly larger number of coincidences (279) than for the POAM III comparison 
( $\sim 4 \mathrm{~h} / 92$ coincidences). In total we found 72 coincident MIPAS profiles for 54 SAGE profiles using miss-time and miss-distance criteria of $<4 \mathrm{~h}$ and $<400 \mathrm{~km}$, respectively. There are quite a number of SAGE PSC profiles with two nearby MIPAS profiles (18). These "double-coincidences" frequently show one PSC and one non-PSC event for MIPAS (11), although the PSC events are not necessarily those with the better miss-time and/or miss-distance match. One possibility is that the result of coincidences might be sensitive to the viewing geometry with respect to each other. In total, we found a corresponding MIPAS PSC coincidence for $38(70 \%)$ of the 54 SAGE PSC profiles. The overall statistic show a slightly better agreement than for the POAM III comparison, which might be a consequence of the latitudes of the coincidences (optical thickness of the PSCs, homogeneity of PSC fields), the viewing geometries, and the miss-time and miss-distance values for the SAGE-MIPAS coincidences which are slightly better than for POAM-MIPAS. The number of PSC events not detected by MIPAS (34) is now smaller than the number of corresponding cloudy profiles (38).

The SAGE III instrument reports a generally higher CTH than MIPAS. The mean difference in CTH is $3 \mathrm{~km}$, with differences of up to $5 \mathrm{~km}$ for single events. We therefore consider detection limits for MIPAS in relation to the extinctions measured by SAGE III. A good estimate of the vertical optical depth (OD) of a PSC observed by SAGE is the integrated extinction profile in the $1022 \mathrm{~nm}$ channel from $2 \mathrm{~km}$ above the tropopause over all altitudes upwards minus a time-dependent background value of the same quantity of non-cloudy profiles. The analysis shows that for $\mathrm{CI}<4 \mathrm{MI}-$ PAS will detect PSCs with a 1022-nm vertical OD $>0.005$ with a probability of $85 \%$, but with only $50 \%$ probability for $\mathrm{OD}<0.005$. The comparison with lidar measurements showed that MIPAS detected PSC with OD $>0.003$ at $1064 \mathrm{~nm}$. In conclusion, the MIPAS detection method is most sensitive to PSCs with a vertical OD greater than 0.005 . A similar analysis was performed based on the SAGE limb extinctions at $1022 \mathrm{~nm}$ at cloud top height with very similar results. For $\mathrm{CI}<4$, the MIPAS PSC detection method is most sensitive to PSCs with equivalent extinctions $>10^{-3} \mathrm{~km}^{-1}$ at $1022 \mathrm{~nm}$ ( $>80 \%$ probability), it is moderately sensitive between $10^{-3}$ to $10^{-4} \mathrm{~km}^{-1}$ (40\% probability) and is not sensitive to PSCs with extinctions $<10^{-4} \mathrm{~km}^{-1}$. Both estimates should provide helpful information for comparisons with microphysical models where it is usual to estimate the equivalent $1022 \mathrm{~nm}$ extinction and/or optical depth of the modelled PSCs for comparisons with lidars and satellite measurements (e.g. Santee et al., 2003).

Overall, the general good agreement between MIPAS and the two solar occultation experiments gives us confidence that the MIPAS cloud detection works well and provides a large number of events for statistical analyses over whole winter periods. The detection method is limited by the coarse FOV and it seems that PSC events with very small vertical extent $(<\mathrm{FOV})$ and the combination of vertical and optical thickness (e.g. low number densities like NAT rocks) might be difficult to detect with MIPAS.

\subsection{MIPAS and SAGE III PSC type differentiation}

Although there are limitations in the PSC type differentiation scheme for MIPAS outlined in Sect. 3.2, we performed a first comparison with results from the SAGE III instrument. The SAGE differentiation method is described in the previous section. We found a perfect agreement between all MIPAS events classified as RE events and SAGE events where the CTH measurement is classified as a PSC Type 1a (7 coincidences, all in December). However, there are also quite a number of SAGE events classified as Type 1a where no RE could be observed in the coincident MIPAS PSC spectra (e.g. 20 events in December) although PSCs were detected by both. This disagreement can, on the one hand, be caused by the smearing effect by the broad FOV of MIPAS, which hampers the analysis of layered cloud structures (e.g. a NAT cloud above an STS cloud), and vertically very thin clouds, on the other hand. In addition, Mie-scattering calculations have already shown (Spang and Remedios, 2003) that the spectral signature around $820 \mathrm{~cm}^{-1}$ disappears in the extinction spectra for large mean radii $(\mathrm{r} \gg 1 \mu \mathrm{m})$ and in consequence an assignment as RE is not necessarily expected for a PSC dominated by NAT 'rocks' for example. Nevertheless, the very promising agreement of the MIPAS RE events with SAGE PSC Type 1a events provides some further evidence that the RE signature in MIPAS spectra is related to clouds with radiation properties dominated by NAT particles, as inferred by Spang and Remedios (2003) from CRISTA data.

\section{PSC occurrence and distribution over the winter}

The following section gives a detailed overview of the PSC distribution observed by MIPAS between November 2002 and March 2003 in combination with ECMWF analyses of the meteorological situation. The results shown are the output of an automated process in which CI was set to 4. This approach is sufficient to identify major PSC episodes which occurred during the winter of 2002/2003.

\subsection{Meteorological condition of the Arctic winter 2002/2003}

The winter of 2002/2003 was characterised by a very early phase (mid November) of cold temperatures in the polar vortex over a large altitude range in the lower and middle stratosphere (Fig. 6). A first minor warming occurred in the upper stratosphere in late December. However, in the lower stratosphere the temperature distribution was scarcely disturbed and the vortex was very strong and symmetric during the entire month of December until the first week in January. Afterwards strong wave activity shifted the vortex core over northern and central Europe (15 January) before - caused 


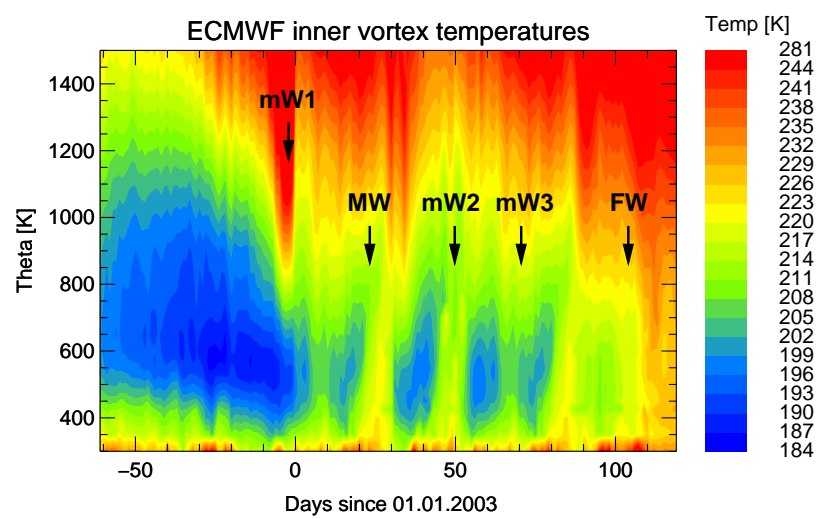

Fig. 6. Inner vortex temperature evolution from 1 November 2002 to 30 April 2003 based on ECMWF analyses. Vortex boundary criterion by Nash et al. (1997) was used. Dates of the minor, major warming ( $\mathrm{mW}$ and MW, respectively) and final warming (FW) events are marked by arrows.

by a major warming - a vortex split happened on 20 January. Reunified, the vortex started to cool again, however it never reached the very low temperatures of the December period. Following a second minor warming the vortex split again around 20 February. Subsequent reunification of the vortex was followed by a period of only moderately cold temperatures in the last week of February and a third minor warming at the beginning of March (day 65). A final period of cold temperatures occurred before a warming of the entire stratosphere took place (>day 90). The vortex degraded slowly but still survived with temperatures generally too high for PSC formation until the final warming occurred in mid April (further details in Naujokat and Grunow, 2003).

\subsection{Temporal evolution}

MIPAS detected around 500 profiles with clear indications of PSCs in the November to March period 2002/2003. Although the coverage (temporal and spatial) of the MIPAS observations during winter 2002/2003 was not continuous due to data gaps in the NRT Level $1 \mathrm{~b}$ data from ESA, days with no data or with only limited observations north of $55^{\circ} \mathrm{N}$ are quite infrequent (gaps in the grey bars in Fig. 7a and b) and the statistical significance of the count statistics (number of PSC events) is high. The PSC activity shown in Fig. 7a as simple count statistics and in Fig. $7 \mathrm{~b}$ as occurrence frequencies can be separated into three phases.

Phase A: The whole of December was characterised by high PSC activity. In late November sporadic PSC events with $\mathrm{CI}<4$ already observed ( 1 event per day) before 1 December showed the first initiation of greater PSC activity followed by a strong increase in activity, reaching the highest occurrence rates of the whole winter on 6 December (nearly $30 \%$ of all MIPAS profiles north of $55^{\circ} \mathrm{N}$ on that day). Until 25 December occurrence rates were at a lower but still high

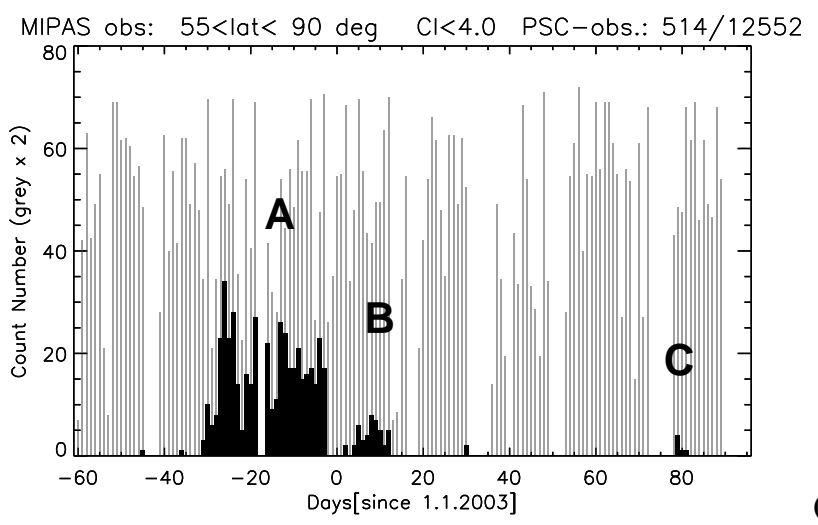

(a)

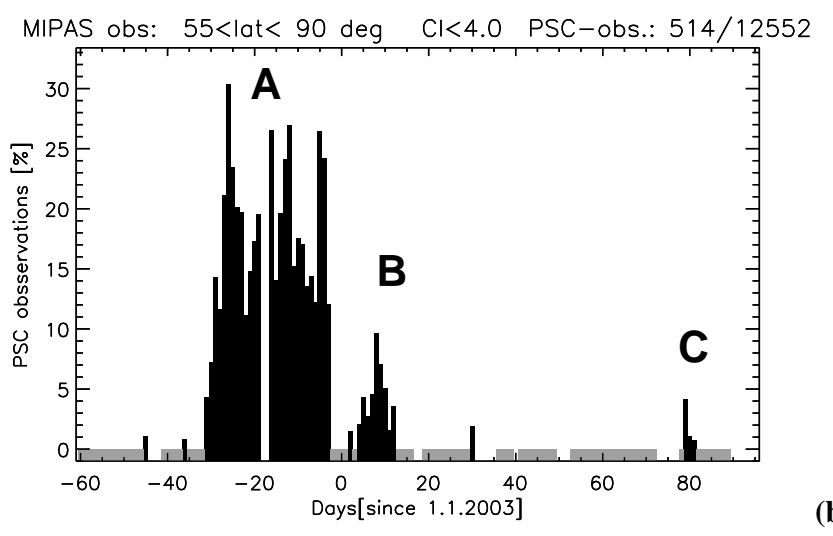

(b)

Fig. 7. PSC count statistics and occurrence frequencies: (a) PSC count statistics (black bars) with respect to the total number (grey bars: to be scaled by factor 2) of observations and (b) PSC occurrence frequency in percentages. All statistics are for north of $55^{\circ} \mathrm{N}$ and running from 1 November to 31 March.

level of around $15-20 \%$. The apparent day-to-day variability in the occurrence rate is mainly caused by a variation in the number of MIPAS observations inside the cold pole of the polar vortex. The PSC activity decreased rapidly in the fourth week of December with final PSC observations on 29 December.

Phase B: After a short warming of polar vortex in the first days of January - during which time no PSCs with $\mathrm{CI}<3$ were observed (30 December-4 January) - weak PSC activity started from 5 January onwards, strengthened gradually and reached a peak between 9 January to 13 January when PSCs were observed by MIPAS in the vicinity of the UK. The occurrence frequencies were significantly lower than in phase A and on 13 January the last strong PSC of this main episode was observed.

Phase C: In February and March, only sporadic PSC events could be observed, mostly with cloud profiles in the domain of $3<\mathrm{CI}<4$. On 21 and 22 March a last phase of cloud observations occurred at low altitudes (Tilmes et al., 2003). These observations are consistent with some localised 

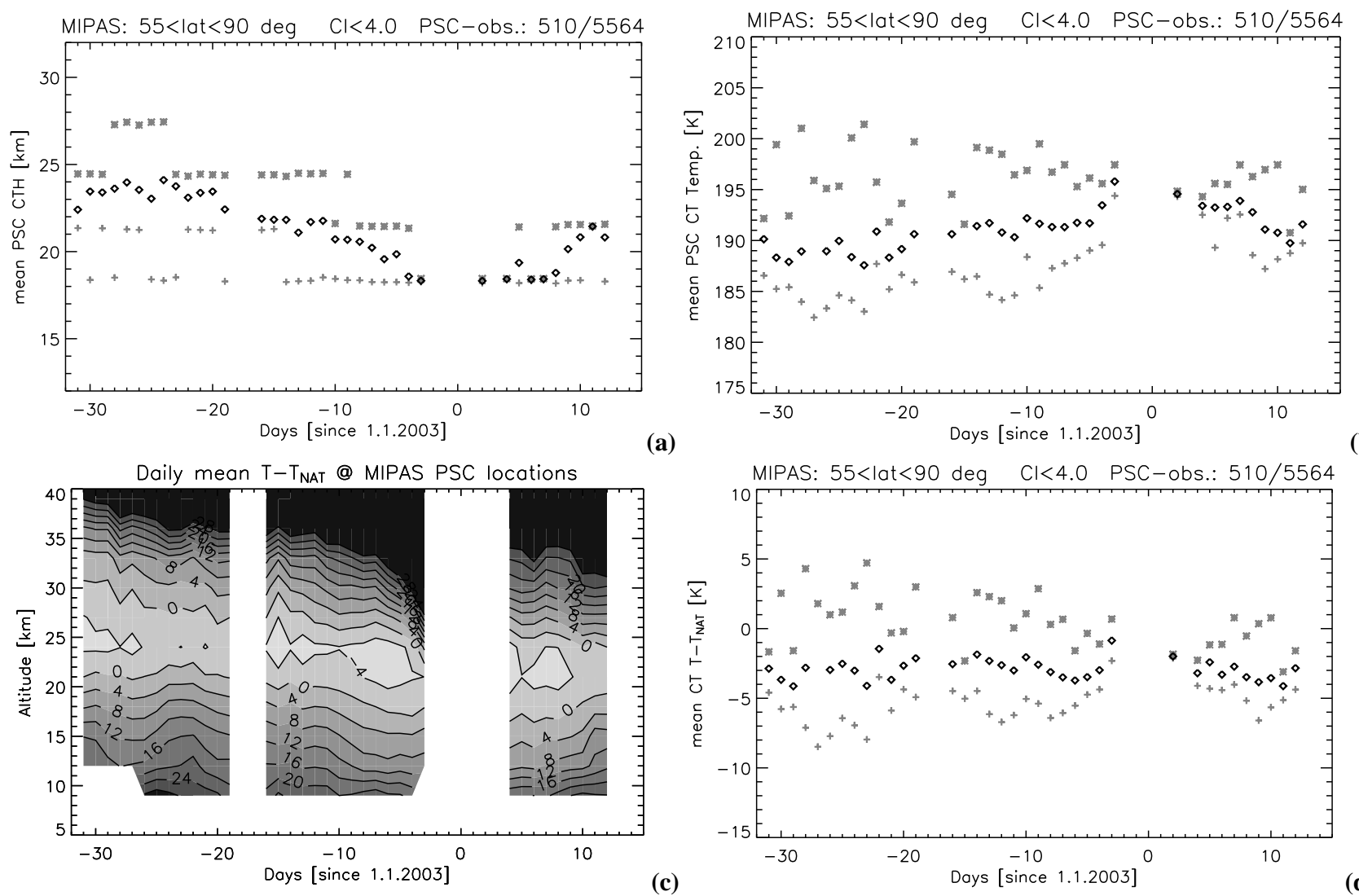

(b)

(c)

Fig. 8. Temporal evolution on a daily basis during the main PSC activity phase December 2002 to mid January 2003 for cloud top parameter: (a) mean cloud top height, (b) mean PSC cloud top temperature based on ECMWF temperatures and (d) mean CTT minus $\mathrm{T}_{N A T}$ (minimum/maximum values for each day are superimposed by grey symbols), and (c) the mean ECMWF temperature profiles minus $\mathrm{T}_{N A T}$ (calculated with constant $\mathrm{HNO}_{3}=10$ ppmv and $\mathrm{H}_{2} \mathrm{O}=5$ ppmv for the entire height range) at MIPAS profile locations.

small areas of $\mathrm{T}<\mathrm{T}_{N A T}$, despite the mean temperature of the polar vortex already being relatively high due to the number of minor warming events. The POAM data also show a few PSC profiles in March, but no coincidences between the two instruments could be found for these profiles. The PSC observations are all observed around $65^{\circ}$ latitude and are unfortunately out of the latitude coverage of the SAGE instrument for this part of the season.

It should be noted that on several days in late November (16 November onwards) MIPAS measured a number of CI profiles in the areas of $\mathrm{T}<\mathrm{T}_{N A T}$ with no indications of PSCs although the POAM and SAGE instruments observed a few profiles with very low extinction coefficients (these profiles were not coincident measurements with MIPAS). This is not necessarily an inconsistency. Steele et al. (2002) already showed for air parcel trajectories that the existence of NAT condensation temperatures, or temperatures below this, for periods of a day or more do not necessarily initiate freezing. Further investigations of MIPAS data with more sensitive CI thresholds need to be performed for this time period and are under way.
Figure 8 shows daily mean CTH in relation to the evolution of mean ECMWF temperature for the main activity phases A and B (1 December-15 January). In the early period, a substantial number of PSCs had cloud top heights in the MIPAS observations in the region of $25 \mathrm{~km}$ and a few extended to even higher altitudes (6/7 December, Fig. 8a). This is consistent with very low mean inner vortex temperatures $(<190 \mathrm{~K})$ up to an isentropic layer around $700 \mathrm{~K}$ in Fig. 6.

After the first phase (1-10 December) of nearly constant mean CTHs, the values decreas to $16 \mathrm{~km}$ until the end of phase A. Over the same period the ECMWF temperature at the tops of the clouds (CTT) increases by around $5 \mathrm{~K}$. This is caused by two effects: First, the cold temperatures of the vortex slowly move down to lower altitudes, which is illustrated by the evolution of the mean T- $\mathrm{T}_{N A T}$ at the PSC profile location (Fig. 8c). And secondly, at the same time a warming of the entire vortex took place, which resulted in a cut in PSC activity at the end of December. This is consistent with the fact that the mean CTT only reaches the NAT existence temperature with the last observations of PSCs at end of phase A (Fig. 8d). It should be noted that the large daily maximum 


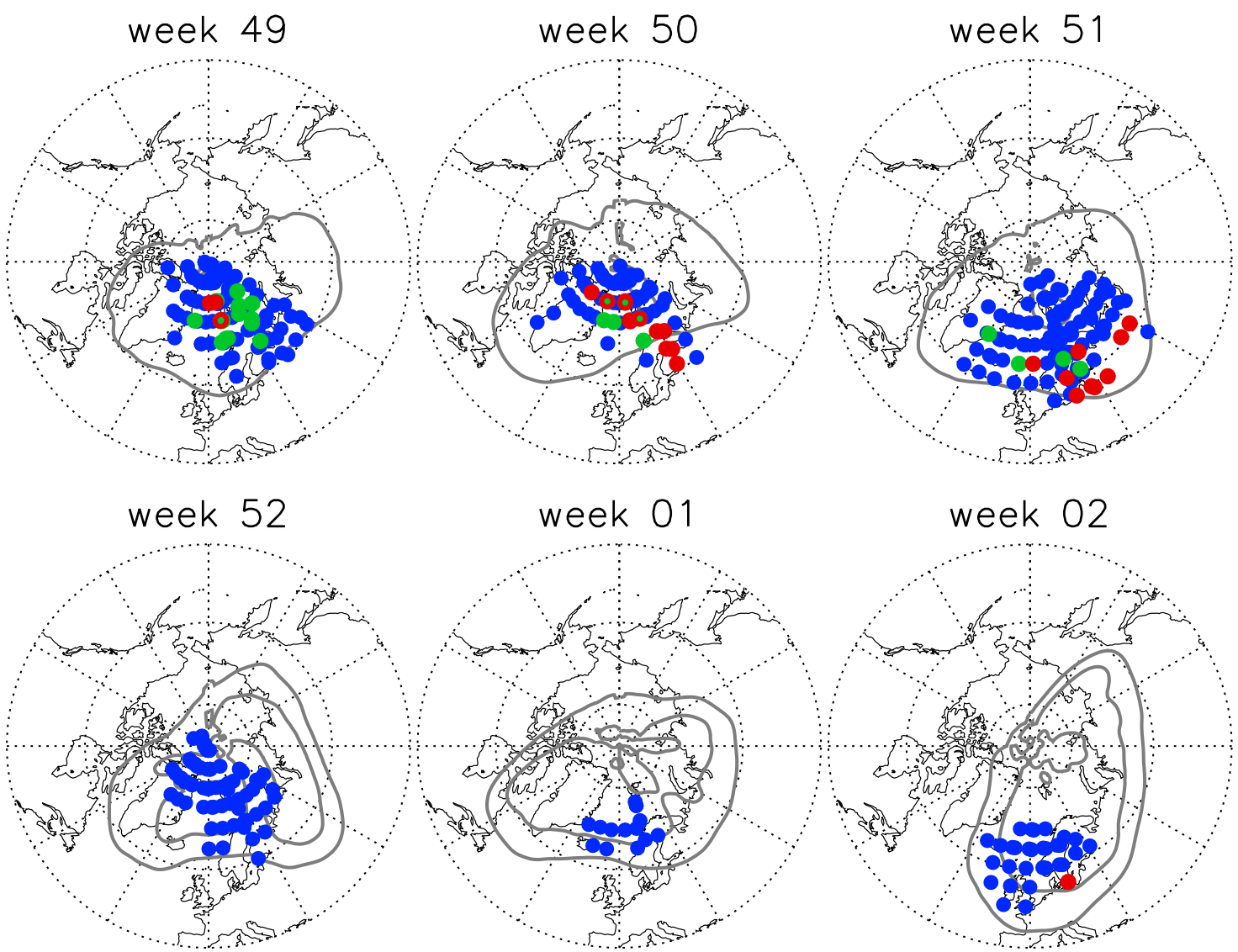

Fig. 9. Temporal evolution of the PSC events with (most likely NAT /red) and without a strong radiance enhancement at $820 \mathrm{~cm}^{-1}\left(\mathrm{presum}^{-}\right.$ ably STS and/or mixed type clouds/in blue) shown for the last four weeks of 2002 (week 49-52) and first two weeks in January 2003 (week 1-2) in the latitude range $40^{\circ}$ to $90^{\circ} \mathrm{N}$. Green dots highlight events with $\mathrm{CI}<1.5$ which refers to events with large optical thickness. The grey contours represent the 60 and $80 \mathrm{PVU}$ contour for midweek conditions at $550 \mathrm{~K}$.

temperature differences with respect to $\mathrm{T}_{N A T}$ (grey asterisks) might be caused by uncertainties in the corresponding temperature profile (ECMWF profile at noon) and the assumption of constant $\mathrm{HNO}_{3}$ and $\mathrm{H}_{2} \mathrm{O}$ values of $10 \mathrm{ppbv}$ and 5 ppmv, respectively, for all altitudes.

To some extent the decreasing CTHs can potentially be caused by denitrification. Irreversible removal of $\mathrm{HNO}_{3}$ by sedimentation of large PSC particles will effectively reduce the $\mathrm{T}_{N A T}$ threshold temperatures. Indications of denitrificaton and corresponding reduction in $\mathrm{T}_{N A T}$ were found in a comparison between model runs for winter $2002 / 2003$ by the denitrification model DLAPSE (Carslaw et al., 2002) and the Ny-Alesund lidar. Towards the end of phase A, in the levels above $19.5 \mathrm{~km}$ the model predicts a $1 \mathrm{~K}$ reduction in $\mathrm{T}_{N A T}$ above $\mathrm{Ny}$-Alesund due to the denitrification in the levels above during the previous weeks (K. Carslaw and G. Mann, University of Leeds, personal communication), which corresponds to the reduction in the PSC top heights deduced from the lidar data. Denitrification was also observed in the MIPAS $\mathrm{HNO}_{3}$ data and will be analysed in detail in a another paper. However, we cannot observe the corresponding decrease in NAT existence temperatures and in mean CTT minus $\mathrm{T}_{N A T}$ Fig. 3d) within the scatter of the data. At the beginning of phase $\mathrm{B}$, the CTHs remained at low altitudes $(18 \mathrm{~km})$ before they started to increase, but simultaneously the height range for temperatures of NAT existence shrank and in consequence the PSC activity died out.

\subsection{Geographical distribution}

In the following section we will give a short summary of the geographical PSC distribution over the winter, which is shown in Fig. 3 and Fig. 9. A more detailed picture is given on the homepage of the EU framework 5 project MAPSCORE (http://www.leos.le.ac.uk/mapscore; link to NRT data), where daily maps of CTH are available throughout the whole of winter 2002/2003. In phase A, the region of PSC activity stretched from the north of Greenland to northern Scandinavia. By 5 December, the region of PSC formation extended to the North Pole and into northern Russia. This early December period, as well as a second period in midDecember, was unusually cold enough for the formation of 

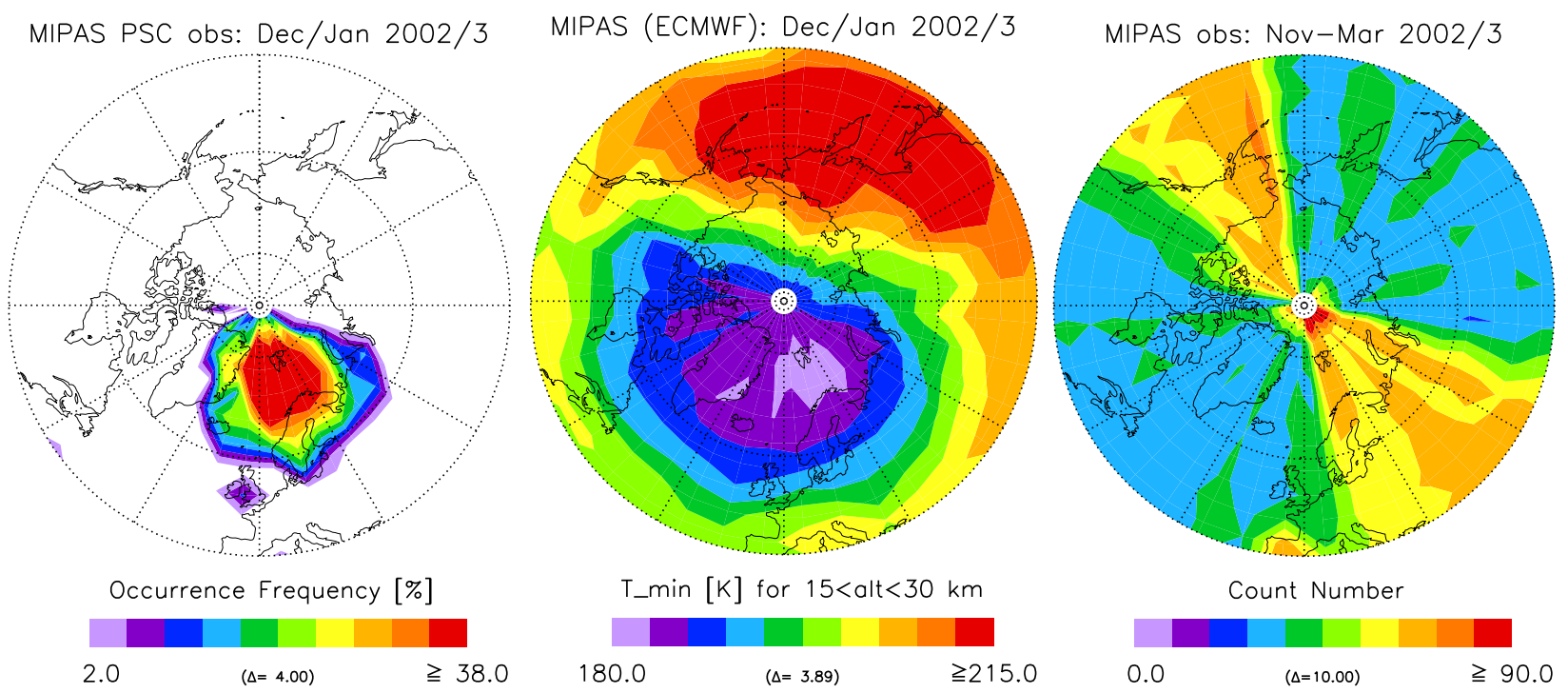

Fig. 10. (a) Occurrence frequency of the MIPAS PSC observations for the time period December-January for $10^{\circ} \times 5^{\circ}$ longitude-latitude grid boxes and (b) the minimum ECMWF temperatures sampled by the MIPAS measurement locations in the 15 to $30 \mathrm{~km}$ height range. (c) Total number of observations in the same grid boxes for November 2002 to March 2003.

water ice clouds. However the area of $\mathrm{T}<\mathrm{T}_{I C E}$ was rather small (not shown) compared to the large region of $\mathrm{T}<\mathrm{T}_{N A T}$ (Fig. 3a).

From 12 December, PSCs were only observed towards the pole and were observed only sporadically above northern Scandinavia. However by 16 December PSCs again appeared over northern Scandinavia and by 17 December had penetrated to southern Greenland and southern Scandinavia. This episode of lower latitude PSC formation lasted until 23 December when the PSCs were observed northwards again for nearly two weeks. In early January the polar vortex was strongly elongated towards the UK and centred over the North Sea. Although the PSC episode did not appear to be as intensely cold as earlier in December, nonetheless a substantial region of PSCs did form at a significantly southerly location in this period (for example traceable over England and south Ireland, Figs. 3, 9f and 10).

The geographical distribution is summarized in Fig. 10 by the polar projection of the PSC occurrence frequencies computed for $10^{\circ} \times 5^{\circ}$ longitude-latitude boxes for the DecemberJanuary period. The highest occurrence rates were found north of Scandinavia up to the pole. This area is coincident and consistent with the area where the lowest minimum temperatures $\left(\mathrm{T}_{\min }\right)$ occurred during the December-January period. The minimum temperatures of Fig. $10 \mathrm{~b}$ are based on ECMWF analyses sampled with MIPAS profile locations in the height range $15-30 \mathrm{~km}$. All occurrence statistics may be slightly biased by the inhomogeneous measurement net of MIPAS, which is illustrated in Fig. 10c by the total count number per grid box. Obviously not all regions of the cold area are sampled with the same density. In consequence, the occurrence frequencies, for example, over Newfoundland, Greenland and Iceland, where low temperatures also occurred, might be underestimated.

\subsection{Temporal and geographical evolution of PSC types}

The temporal and spatial evolution of the RE events or NAT cloud type indicator for MIPAS (Sect. 4.3) is shown in Fig. 9 for the key PSC phases of the winter (phases A and B). In the first three weeks of observations in December 2002, a change in the occurrence of RE events is obvious. In the first week (starting 2 December) only a few events with a significant enhancement could be observed (red symbols). In the second week, the number of observed RE events increased, followed by a slight decrease in the activity during the third week, before any indications of RE events disappeared in the last week of December and first week of January. At first sight, it appears that the RE events are linked to the episode of the coldest temperatures in the polar vortex (mid-December). However, the mean temperature difference $\left(\mathrm{T}-\mathrm{T}_{N A T}\right)$ for RE and NRE events shows no obvious indications of this hypothesis, and only an insignificant tendency for warmer temperatures for RE events $(-2.9 \mathrm{~K})$ than for NRE events $(-3.0 \mathrm{~K})$. A separation in $\mathrm{T}^{-\mathrm{T}_{N A T}}$ between RE events (NAT clouds) and non-optically thick NRE events (STS clouds) is shown by Spang and Remedios (2003) for the CRISTA Antarctic observations. However, the relatively low number of cloud events for this northern hemisphere winter compared to the CRISTA observations in the SH prevents a proper comparison. Likewise the subset of RE events for this winter is much smaller $(<20 \%)$ than in the CRISTA analyses 
( $>60 \%$ ), which might refer to some fundamental differences between the formation of PSC in the two hemispheres.

A further possibility is that mixed-type cloud might be present in the majority of the northern hemisphere observations, where the temperatures are closer to PSC threshold temperatures and air parcel trajectories cross these thresholds more frequently than in the southern hemisphere. Indication for this effect can be found in the lidar data, where many layered structures of the PSC types were found ("sandwich structures"). Mixed type clouds in the FOV of the instrument make any separation and classification of cloud type events more difficult and will produce enhanced scatter in any statistical analysis.

Figure 9 additionalls shows events with a significantly high optical depth $(\mathrm{CI}<1.5$, optically thick cloud: $\mathrm{CI}$ close to one, e.g. Spang and Remedios, 2003). No extreme events with $\mathrm{CI}<1.2$ were detected and only a few events with very high optical depth could be found $(1.2<\mathrm{CI}<1.5)$. The latter were detected only in the first three weeks of PSC sightings (Phase A). This is in agreement with the vortex evolution, with temperatures mostly well above the ice saturation temperature $\mathrm{T}_{I C E}$ and the fact that an optical thickness close to one can only be produced by ice or in specific cases by STS clouds (M. Höpfner, IMK, Research Centre Karlsruhe, personal communication). Events with $1.2<\mathrm{CI}<1.5$ can be related to clouds with large particles $(\mathrm{r}>1 \mu \mathrm{m})$ and in consequence to enhanced radiation by scattering processes (e.g. ice or NAT), and/or clouds with a large volume density of the particles (e.g. a very dense STS cloud). The lack of observations of low CI events throughout January and the rest of the winter is in agreement with the higher vortex temperatures with respect to $\mathrm{T}_{N A T}$ for this part of the winter.

\section{Conclusions}

The cloud detection method developed for CRISTA has been successfully implemented for MIPAS. The method provides a fast and simple technique for retrieving spatial location and altitude information (cloud top height) of polar stratospheric clouds. The PSC detection scheme with a threshold value of $\mathrm{CI}<4$, was validated, based on comparisons with lidar and satellite data. The agreement in the detection of PSC events between MIPAS and the lidars is excellent, which gives confidence that the method can be applied to multi-year analyses. The PSC CTHs and locations inferred from MIPAS are also in good agreement with POAM III extinction analyses. However, the occultation measurements, especially SAGE III, potentially show a higher sensitivity for the detection of optically thinner clouds than the equivalent $10^{-3} \mathrm{~km}^{-1}$ limit $(1022 \mathrm{~nm})$ for $\mathrm{CI}<4$ and, in consequence, display apparently higher CTHs.

For the first time, coverage of PSC evolution could be examined throughout the Arctic polar vortex up to the pole on many days during the winter period. Winter 2002/2003 was analysed in detail and can be characterised in three phases: (1) A very intense and unusual early cold phase in the first three weeks of December with high PSC activity and partly optically thick cloud events. This phase is also characterised by a reduction in the mean CTH consistent with a descent of the height of the vertical temperature minimum in the polar vortex. (2) After a slight warming of the polar vortex temperatures were again cold enough between 5 to 13 January for a phase of more moderate PSC activity. Nonetheless, PSCs were spread extremely far towards mid latitudes, down to the U.K. and the south coast of Ireland. (3) A few PSC profiles were observed in mid February $(3>\mathrm{CI}>4)$ and around 20 March when the vortex - already warmer after two major warming events and vortex splits - was reunified again. The observations are consistent with the last small areas of $\mathrm{T}<\mathrm{T}_{N A T}$ deduced from ECMWF analyses.

A number of MIPAS PSC spectra show the same distinctive spectral feature around $820 \mathrm{~cm}^{-1}$ as found previously for CRISTA data in the Antarctic. The spatial distribution of these RE events was examined through winter 2002/2003 as they are expected to indicate NAT particles, albeit with mean particle sizes less than $2-3 \mu \mathrm{m}$ radius. The subset of spectra is considerably smaller than for CRISTA and so far no significant temperature separation between NRE and RE events could be found. The main occurrence of the NAT particles is seen to take place in December and not in January when a second phase of PSC formation was observed. Further studies with microphysical and denitrification models would be interesting to examine the apparent differences in NAT formation in phases of winter 2002/2003 and the differences between Arctic and Antarctic winters.

Acknowledgements. The work presented in this paper was supported by the EU framework 5 project MAPSCORE, EVK2CT-2000-00072. We would like to thank ESA for the early delivery of preliminary MIPAS Level $1 \mathrm{~b}$ and Level 2 data as part of AO357 proposal CUTLSOM. The authors acknowlege the helpful comments by the two reviewers.

Edited by: K. Carslaw

\section{References}

Bevilacqua, R. M., Fromm, M. D., Alfred, J. M., et al.: Observations and analysis of polar stratospheric clouds detected by POAM III during the 1999/2000 Northern Hemisphere winter, J. Geophys. Res., 107(D20), 8281, doi:10.1029/2001JD000477, 2002.

Browell, E. V., Butler, C. F., Ismail, S., Robinette, P. A., Carter, A. F., Higdon, N. S., Toon, O. B., Schoeberl, M. R., and Tuck, A. F.: Airborne lidar observations in the wintertime Arctic stratosphere: polar stratospheric clouds, Geophys. Res. Lett., 17, 385388, 1990.

Carli, B., Alpaslan, D., Carlotti, M., et al.: First results of MIPAS/ENVISAT with operational Level 2 code, Adv. Space Res., 33, 1012-1019, 2004. 
Carslaw, K. S., Kettleborough, J., Northway, M. J., Davies, S., Gao, R.-S., Fahey, D. W., Baumgardner, D. G., Chipperfield, M. P., and Kleinböhl, A.: A Vortex-Scale simulation of the growth and sedimentation of large nitric acid hydrate particles, J. Geophys. Res., 107, 8300, doi:10.1029/2001JD000467, 2002.

Fahey, D. W., Gao, R. S., Carslaw, K. S., et al.: The detection of large $\mathrm{HNO}_{3}$-containing particles in the winter Arctic stratosphere, Science, 291, 1026-1031, 2001.

Fischer, H. and Oelhaf, H.: Remote sensing of vertical profiles of atmospheric trace constituents with MIPAS limb emission spectrometers, Appl. Opt., 35(16), 2787-2796, 1996.

Fromm, M., Alfred, J., and Pitts, M.: A unified, long-term, highlatitude stratospheric aerosol and cloud database using SAM II, SAGE II, and POAM II/III data: Algorithm description, database definition, and climatology, J. Geophys. Res., 108(D12), 4366, doi:10.1029/2002JD002772, 2003.

Höpfner, M., Oelfhaf, H., Wetzel, G., et al.: Evidence of scattering of tropospheric radiation by PSCs in mid-IR emission spectra: MIPAS-B observations and KOPRA simulations, Geophys. Res. Lett., 29(8), doi:10.1029/2001GL014443, 2002.

Höpfner, M.: Study on the impact of polar stratospheric clouds on high resolution mid-IR limb emission spectra, J. Quant. Spec. Rad. Trans., 83, 93-107, 2004.

Nash, E. R., Newman, P. A., Rosenfield, J. E., and Schoeberl, M. R.: An objective determination of the polar vortex using Ertel's potential vorticity, J. Geophys. Res., 101, 9471-9478, 1997.

Naujokat, B. and Grunow, K.: The stratospheric arctic winter 2002/03: Balloon flight planning by trajectory calculations. Proceedings of the 16th ESA Symposium on European Rocket and Balloon Programmes and Related Research, St. Gallen 2003 (ESA SP-530), 421-425, 2003.

Mann, G. W., Davies, S., Carslaw, K. S., and Chipperfield, M. P.: Factors controlling Arctic denitrification in cold winters of the 1990s, Atmos. Chem. Phys., 3, 403-416, 2003,

\section{SRef-ID: 1680-7324/acp/2003-3-403.}

Massie, S. T., Baumgardner, D., and Dye, J. E.: Estimation of polar stratospheric cloud volume and area densities from UARS, SAGE II, and POAM II extinction data, J. Geophys. Res., 103, 5773-5783, 1998.

McCormick, M. P., Steel, H. M., Hamill, P., Chu, W. P., and Swissler, T. J.: Polar stratospheric cloud sightings by SAM II, J. Atmos. Terr. Phys., 39, 1387-1397, 1982.

Peter, Th.: Microphysics and heterogeneous chemistry of polar stratospheric clouds, Annu. Rev. Phys. Chem., 48, 785-822, 1997.

Poole, L. R., Trepte, C. R., Harvey, V. L., Toon, G. C., and van Valkenburg, R. L.: SAGE III observations of Arctic polar stratospheric clouds - December 2002, Geophys. Res. Lett., 30(23), 2216, doi:10.1029/2003GL018496, 2003.

Riese, M., Spang, R., Preusse, P., Ern, M., Jarisch, M., Offermann, D., and Grossmann, K. U.: Cryogenic Infrared Spectrometers and Telescopes for the Atmosphere (CRISTA) data processing and atmospheric temperature and trace gas retrieval, J. Geophys. Res., 104, 16349-16367, 1999.

Remedios J. J. and Spang, R.: Detection of cloud effects in MIPAS spectral data and implications for the MIPAS operational processor, Proceedings of the ENVISAT Calibration Review, 913 September 2002, ESA-ESTEC, Noordwijk, The Netherlands, 2002.
Remedios, J. J. and Spang, R.: MIPAS observations of clouds and their effects on Level 2 trace gas products, Proceedings of the ENVISAT Validation Workshop, 9-13 December 2002, ESAESRIN, Frascati, Italy, 2003.

Santee, M. L., Tabazadeh, A., Manney, G. L., Fromm, M. D., Bevilacqua, R. M., Waters, J. W., and Jensen, E. J.: A Lagrangian approach to studying Arctic polar stratospheric clouds using UARS MLS HNO3 and POAM II aerosol extinction measurements, J. Geophys. Res., 107(D10), 4098, doi:10.1029/2000JD000227, 2002.

Spang, R. and Remedios, J. J.: Observations of a distinctive infrared spectral feature in the atmospheric spectra of polar stratospheric clouds measured by the CRISTA instrument, Geophys. Res. Lett., 30(16), 1875, doi:10.1029/2003GL017231, 2003.

Spang, R., Riese, M., and Offermann, D.: CRISTA-2 observations of the south polar vortex in winter 1997: A new dataset for polar process studies, Geophys. Res. Lett., 28, 3159-3162, 2001.

Spang, R., Eidmann, G., Riese, M., Offermann, D., Preusse, P., Pfister, L., and Wang, P. H.: CRISTA observations of cirrus clouds around the tropopause, J. Geophys. Res., 107(D23), 8174, doi:10.1029/2001JD000698, 2002.

Spang, R., Remedios, J. J., and Barkley, M. P.: Colour indices for the detection and differentiation of cloud types in infra-red limb emission spectra, Adv. Space Res., 33, 1041-1047, 2004.

Steele, H. M., Lumpe, J. D., Turco, R. P., Bevilacqua, R. M., and Massie, S. T.: Role of temperature history in polar stratospheric clouds sightings, J. Geophys. Res., 107(D22), 4618, doi:10.1029/2001JD001261, 2002.

Strawa, A. W., Drdla, K., Fromm, M., Pueschel, R. F., Hoppel, K. W., Browell, E. V., Hamill, P., and Dempsey, D. P.: Discriminating Types Ia and Ib polar stratospheric clouds in POAM satellite data, J. Geophys. Res., 107(D20), 8291, doi:10.1029/2001JD000458, 2002.

Tabazadeh, A., Jensen, E. J., Toon, O. B., Drdla, K., and Schoeberl, M. R.: Role of the stratospheric freezing belt in denitrification, Science, 291, 2591-2594, 2001.

Taylor, F. W., Lambert, A., Grainger, R. G., Rodgers, C. D., and Remedios, J. J.: Properties of northern hemisphere polar stratospheric clouds and volcanic aerosol in 1991/92 from UARS/ISAMS satellite measurements, J. Atmos. Sci., 51, 20, 3019-3026, 1994.

Tilmes, S., Müller, R., Grooß, J.-U., Höpfner, M., Toon, G. C., and Russell III, J. M.: Very early chlorine activation and ozone loss in the Arctic winter 2002-2003, Geophys. Res. Lett., 30(23), 2201, doi:10.1029/2003GL018079, 2003.

Voigt, Ch., Schreiner, J., Kohlmann, A., et al.: Nitric acid trihydrate (NAT) in polar stratospheric clouds, Science, 290, 1756-1758, 2000.

von Clarmann, T., Glatthor, N., Grabowski, U., et al.: Retrieval of temperature and tangent altitude pointing from limb emission spectra recorded from space by the Michelson Interferometer for Passive Atmospheric Sounding (MIPAS), J. Geophys. Res., 108(D23), 4736, doi:10.1029/2003JD003602, 2003.

von Zahn, U., von Cossart, G., Fiedler, J., Fricke, K. H., Nelke, G., Baumgarten, G., Rees, D., Hauchecorne, A., and Adolfsen, K.: The ALOMAR Rayleigh/Mie/Raman lidar: objectives, configuration, and performance, Ann. Geophys., 18, 815-833, 2000, SRef-ID: 1432-0576/ag/2000-18-815. 